\title{
Linearized Theory of a Partially Cavitating Plano-Convex Hydrofoil Including the Effects
} of Camber and Thickness

By R. B. Wade'

\author{
HYDRODYNAMICS LABORATC
CALIFORNIA INSTITUTE OF TECHNOLOG: \\ 1201 E. CALIFORNIA STREET \\ PASADENA 4, CALIFORNIA
}

\begin{abstract}
The linearized treatment of the flow over a partially cavitating single hydrofoil having a flat pressure surface and a circular-arc suction side is presented. The flow is treated as a two-dimensional, steady, inviscid flow. Further assumptions made are those of incompressibility and irrotationality. The results obtained are compared with experiment and generally good correlation is found for the ranges of validity of the linearization.
\end{abstract}

\section{Introduction}

THE investigation of the behavior of hydrofoils under varying degrees of cavitation has been the object of a great deal of interest. Several theoretical approaches have been developed for treating these problems both from a nonlinear and linearized point of view.

From experimental observation [1],2 it has been established that the cavity flow around a hydrofoil may be divided into three distinct regimes: The partially cavitating region, the fully cavitating region, and an inherently unsteady zone connecting these two flow configurations. The partially cavitating region is associated with flows where the cavity length is less than the chord

${ }^{1}$ Research Fellow, Division of Engineering and Applied Science, California Institute of Technology, Pasadena, Calif.

${ }^{2}$ Numbers in brackets designate References at end of paper.

Manuscript received at SNAME Headquarters, November 23, 1965. length of the body and consequently the cavity terminates on the upper (or suction) surface of the foil. In the fully cavitating region, however, the cavity collapses downstream of the trailing edge and the entire upper surface of the foil is enclosed within the cavity.

The problem of fully cavitating flows past arbitrary shaped hydrofoils has been thoroughly treated by Wu and Wang using nonlinear techniques [2] and by many authors using linearized methods [3]. For the case of the partially cavitating body, however, relatively few results have been published. In this region of flow where the camber and thickness of the profile play a role linearized techniques are much more amenable to solution than are nonlinear methods owing to the difficulty of choosing a suitable model for representing the flow in this latter case. Wu [4] has worked out the case of a partially cavitating flat plate using a nonlinear method but all other solutions have been obtained using the linearized technique. These solutions include that of the

Nomenclature

$$
\begin{aligned}
a & =\text { scale parameter }=\sqrt{\frac{1-l}{l}} \\
A, B & =\text { constants } \\
C_{L} & =\text { lift coefficient }=\frac{L}{\rho V^{2} c / 2} \\
c & =\text { chord length } \\
G & =\text { complex function }=\frac{W(\zeta)}{H(\zeta)} \\
H & =\text { homogeneous solution }=\sqrt{\zeta(\zeta-1)} \\
I & =\text { integral } \\
i & =\sqrt{-1} \\
K & =\text { cavitation number }=\frac{p_{\infty}-p_{c}}{\rho V^{2} / 2} \\
l & =\text { cavity length } \\
p_{\infty} & =\text { pressure at infinity }
\end{aligned}
$$

$$
\begin{aligned}
p_{c} & =\text { cavity pressure } \\
P & =\text { rational complex function }=\frac{A \zeta+B}{\zeta(\zeta-1)} \\
R & =\text { radius } \\
u, v & =\text { velocity components in } x, y \text {-directions } \\
u^{\prime}, v^{\prime} & =\text { perturbation components } \\
V & =\text { upstream velocity } \\
W(z) & =\text { complex velocity functions } \\
w(z) & =\text { coordinate axes in physical plane } \\
x, y & =\text { complex physical plane } \\
z=x+i y & =\text { angle of attack } \\
\rho & =\text { density } \\
\Omega & =\text { angle subtended by tangents to upper foil surface } \\
\zeta=\xi+i \eta & =\text { transformed complex plane } \\
\xi, \eta & =\text { coordinate axes in transformed plane }
\end{aligned}
$$


partially cavitating flat plate by Acosta [5] which was also subsequently treated by Geurst [6]. In Geurst's paper the formulation is carried out for an arbitrary profile having zero thickness. The case of a profile including thickness effects has not been treated in the literature.

The present paper deals with the linearized treatment of a partially cavitating flow over a plano-convex (flat pressure surface and circular-are suction side) hydrofoil including the effects of camber and profile thickness. The results so obtained are then compared with the experimental results obtained on such hydrofoils by several authors, viz., Balhan [7], Meijer [8], and Wade [1]. The purpose of studying such a profile section, a member of the Kármán-Trefftz family of airfoils, arises from the extensive use of slight variants from this form in propeller work.

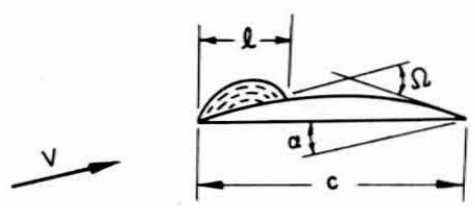

Fig. 1 Partially cavitating plano-convex hydrofoil

\section{Formulation of Problem}

The hydrofoil is held at an angle of attack, $\alpha$, to the free-stream velocity, $V$, as illustrated in Fig. 1. For the present problem it is assumed that a cavity forms on the top side of the hydrofoil starting at the leading edge. The cavity then terminates on the upper surface. The angle subtended by the tangents to the circular surface at the leading and trailing edges, $\Omega$, is assumed to be small as is the angle of attack, $\alpha$. These assumptions are in keeping with the linearized theory [3].

With these assumptions it is possible to consider the velocity field as a perturbation on the free-stream velocity, $V$, allowing one to write the velocity at any point in the fluid as

$$
\bar{q}=V\left(1+u^{\prime}, v^{\prime}\right)=(u, v)
$$

where $u^{\prime}, v^{\prime}$ are the perturbation components. Furthermore the equation for the circular-are suction surface can be written in the form

$$
\frac{d y}{d x}=\frac{\Omega}{2}(1-2 x)
$$

The boundary conditions on the velocity function, within the framework of the linearized theory, then become with the help of Bernoulli's equation

$v=-V \alpha+\frac{V \Omega}{2}(1-2 x)$, for the top wetted surface

$v=-V \alpha$, for the bottom wetted surface

$u=V\left(1+\frac{K}{2}\right)$ on the cavity surface

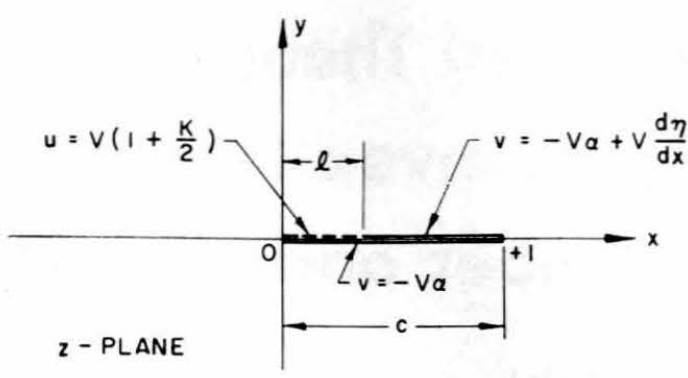

Fig. 2 Linearized problem in physical $z$-plane

$K$ is the cavitation number defined as

$$
K=\frac{p_{\infty}-p_{c}}{\rho V^{2} / 2}
$$

where

$$
\begin{aligned}
& p_{\infty}=\text { pressure at infinity } \\
& p_{c}=\text { cavity pressure }
\end{aligned}
$$

These boundary conditions are applied along a slit representing the body in the physical plane, as illustrated in Fig. 2.

From the initial assumptions that the flow is incompressible and irrotational, the function

$$
W(z)=u-i v
$$

is therefore an analytic function of the complex variable $z$. The transformation

$$
\zeta=i a \sqrt{\frac{z}{z-1}}
$$

where

$$
a=\sqrt{\frac{1-l}{l}}
$$

transforms the slit in the $z$-plane into the upper half $\zeta$ plane, such that the entire real axis of the $\zeta$-plane becomes the surface of the foil. Furthermore the region $0<\zeta$ $<1$ becomes the cavity surface. With this transformation the point at infinity in the $z$-plane is transformed into the point $i a$ in the $\zeta$-plane as seen in Fig. 3 . The relevant boundary conditions are also shown in this figure.

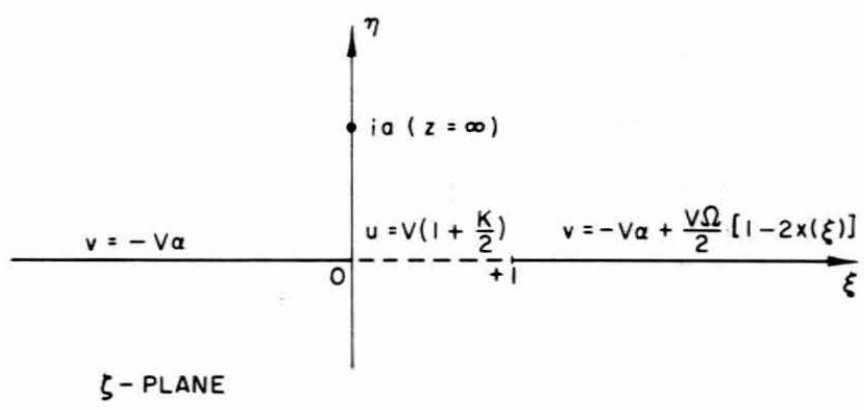

Fig. 3 Transform $\zeta$-plane 
Further conditions to be satisfied are that the velocity at infinity be equal to the free-stream velocity, $V$, i.e.

$$
\lim _{z \rightarrow \infty} W(z)=V
$$

and that the cavity-hydrofoil system form a closed body. This requirement may be expressed as

$$
\int_{\text {body }} d y=0
$$

where $y$ represents the ordinates of points on the bodycavity system.

Furthermore, at the trailing edge, due to the finite trailing-edge angle of the hydrofoil, the velocity there must behave logarithmically. This replaces the usual Kutta condition at the trailing edge.

Considering the function

$$
w(z)=W(z)-V\left(1+\frac{K}{2}\right)-i V \alpha
$$

in the $\zeta$-plane, we have:

$$
\begin{array}{rlrl}
\text { Imaginary part } w & =0 & -\infty<\xi<0 \\
\text { Real part } w & =0 & 0 & <\xi<1 \\
\text { Imaginary part } w & =-\frac{V \Omega}{2}[1-2 x(\xi)] & 1<\xi<\infty
\end{array}
$$

If we continue $w(z)$ analytically through the interval $0<$ $\xi<1$ into the lower half plane, such that

$$
w(\overline{\zeta)}=-\overline{w(\zeta)}
$$

then the real part of $w$ is an uneven function of $\eta$ and the imaginary part of $w$ is an even function of $\eta$. We can thus formulate the following boundary-value problem in the $\zeta$-plane:

$w^{+}+w^{-}=0$

$$
-\infty<\xi<0
$$

$w^{+}-w^{-}=0$

$0<\xi<1$

$w^{+}+w^{-}=-2 i \frac{V \Omega}{2}[1-2 x(\xi)]$

$1<\xi<\infty$

where the superscripts refer to the value of $w(\zeta)$ as $\eta \rightarrow \pm 0$. The problem therefore reduces to a Hilbert problem the solution of which can be found by applying the procedures given in reference [9].

\section{Solution of the Problem}

Let us first consider the homogeneous problem

$$
\begin{array}{rr}
H^{+}+H^{-}=0 & -\infty<\xi<0 \\
H^{+}-H^{-}=0 & 0<\xi<1 \\
H^{+}+H^{-}=0 & 1<\xi<\infty
\end{array}
$$

It can be seen that $H(\zeta)$ is continuous for $0<\xi<1$ but has a jump for $\xi$ outside of this range. A function satisfying these conditions is

$$
H=\sqrt{\zeta(\zeta-1)}
$$

where we take the branch cuts of $H$ to be along the real axis outside the interval $0<\xi<1$, and we further require that

$$
H \sim \zeta \text { as } \zeta \rightarrow \infty
$$

We now consider the function

$$
G(\zeta)=\frac{w(\zeta)}{H(\zeta)}
$$

The boundary conditions for this function are

$$
\begin{aligned}
G^{+}-G^{-}=\frac{w^{+}}{H^{+}}-\frac{w^{-}}{H^{-}}=\left(w^{+}+w^{-}\right) / H^{+} & =0 \\
-\infty<\xi<0 & \\
G^{+}-G^{-}=\frac{w^{+}}{H^{+}}-\frac{w^{-}}{H^{-}}=\left(w^{+}-w^{-}\right) / H^{+} & =0 \\
& 0<\xi<1
\end{aligned}
$$

$$
\begin{aligned}
G^{+}-G^{-}= & \frac{w^{+}}{H^{+}}-\frac{w^{-}}{H^{-}}=\left(w^{+}+w^{-}\right) / H^{+} \\
& =\frac{-i V \Omega}{\sqrt{\xi(\xi-1)}}[1-2 x(\xi)] \quad 1<\xi<\infty
\end{aligned}
$$

By means of Plemelj's formula, we can express an analytic function in the upper half plane by its values along the entire real axis according to the formula

$$
F(z)=\frac{1}{2 \pi i} \int_{-\infty}^{\infty} \frac{f(x)}{x-z} d x
$$

where $f(x)=f^{+}(x)-f^{-}(x)$.

Accordingly, we obtain

$$
G(\zeta)=-\frac{V \Omega}{2 \pi} \int_{1}^{\infty} \frac{1-2 x(\xi)}{\sqrt{\xi(\xi-1)}(\xi-\zeta)} d \xi
$$

This solution represents a particular solution of the problem; the general solution being given by

$$
\begin{array}{r}
w(\zeta)=-\frac{V \Omega}{2 \pi} \sqrt{\zeta(\zeta-1)} \int_{1}^{\infty} \frac{1-2 x(\xi)}{\sqrt{\xi(\xi-1)(\xi-\zeta)}} d \xi \\
+P(\zeta) \sqrt{\zeta(\zeta-1)}
\end{array}
$$

where $P(\zeta)$ is a rational function of $\zeta$ which can have poles only at the points $\xi=0,1$. At the trailing edge, i.e., the point $\zeta=\infty$, in the $\zeta$-plane, $w(\zeta)$ can at most behave logarithmically. This behavior, a consequence of the linearized thickness effect, is already incorporated in the integral part of the solution. Hence $P(\zeta)$ can only be of the form

$$
\frac{A \zeta+B}{\zeta(\zeta-1)}
$$

where $A, B$ are real constants. We finally get for our solution 


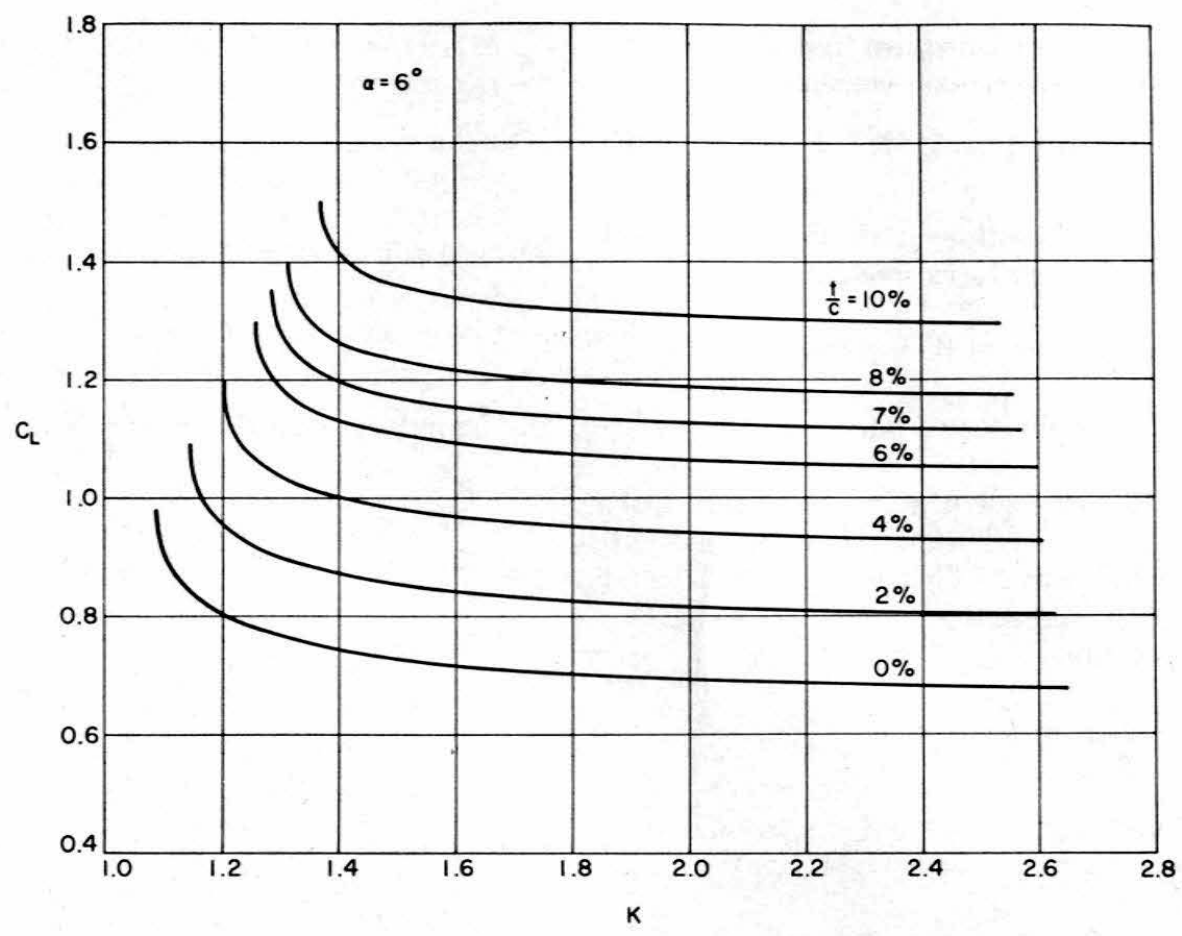

Fig. 4 Lift coefficient versus cavitation number $K$ for various thickness ratios at a fixed angle of attack of $6 \mathrm{deg}$

$$
\begin{array}{r}
w(\zeta)=-\frac{V \Omega}{2 \pi} \sqrt{\zeta(\zeta-1)} \int_{1}^{\infty} \frac{1-2 x(\xi)}{\sqrt{\xi(\xi-1)}(\xi-\zeta)} d \xi \\
\quad+\frac{A \zeta+B}{\sqrt{ } \zeta(\zeta-1)}
\end{array}
$$

where $x(\xi)$ can be obtained from the transformation equation (5a) as

$$
x(\xi)=\frac{\xi^{2}}{\xi^{2}+a^{2}}
$$

The constants $A$ and $B$ can be evaluated from the condition that

$$
W(\zeta)=V \text { at } \zeta=i a
$$

As we will only be concerned with evaluating the foregoing integral at $\zeta=i a$, we can write this integral as

$$
\begin{aligned}
I=\int_{1}^{\infty} \frac{\xi\left(a^{2}-\xi^{2}\right)}{\sqrt{\xi(\xi-1)}\left(\xi^{2}+a^{2}\right)^{2}} d \xi \\
+i a \int_{1}^{\infty} \frac{\left(a^{2}-\xi^{2}\right)}{\sqrt{\xi(\xi-1)}\left(\xi^{2}+a^{2}\right)^{2}} d \xi
\end{aligned}
$$

For purposes of computation it is more convenient to change the limits of integration to a finite interval. By successively substituting $\xi=1 / t$ and $t=1-x^{2}$, these integrals reduce to

$$
I=2 \int_{0}^{1} \frac{\left(a^{2} t^{2}-1\right)}{\left(1+a^{2} t^{2}\right)^{2}} d x+i 2 a \int_{0}^{1} \frac{t\left(a^{2} t^{2}-1\right)}{\left(1+a^{2} t^{2}\right)^{2}} d x
$$

or

$$
I=I_{1}+i I_{2}
$$

where $I_{1}$ and $I_{2}$ are only functions of the parameter $a$. The integrals are represented in this fashion as it simplifies the numerical integration process. It is possible to evaluate these integrals in closed form but this leads to a complicated expression which is not very useful.

The constants $A$ and $B$ can now be evaluated with the aid of equation (6). After some manipulation we obtain

$$
\begin{aligned}
A=-V \frac{\left(1+a^{2}\right)^{1 / 4}}{a^{1 / 2}} & {\left[\frac{K}{2} \sin \frac{\psi}{2}+\alpha \cos \frac{\psi}{2}\right] } \\
+ & \frac{V \Omega}{2 \pi}\left(1+a^{2}\right)^{1 / 2}\left[I_{1} \sin \psi+I_{2} \cos \psi\right]
\end{aligned}
$$

and

$$
\begin{aligned}
B=-V a^{1 / 2}(1+ & \left.a^{2}\right)^{1 / 4}\left[\frac{K}{2} \cos \frac{\psi}{2}-\alpha \sin \frac{\psi}{2}\right] \\
& +\frac{V \Omega}{2 \pi} a\left(1+a^{2}\right)^{1 / 2}\left[I_{1} \cos \psi-I_{2} \sin \psi\right]
\end{aligned}
$$

where $\psi=\pi+\tan ^{-1} 1 / a$. These expressions express the constants $A$ and $B$ in terms of the parameter $a$. The first terms in the aforementioned expressions correspond to the flat-plate solution and the camber and thickness effects are incorporated in the second terms.

The relationship between the cavitation number $K$ and the cavity length $l$ is obtained by applying the closure condition, equation (7). This condition may be written in the following form: 


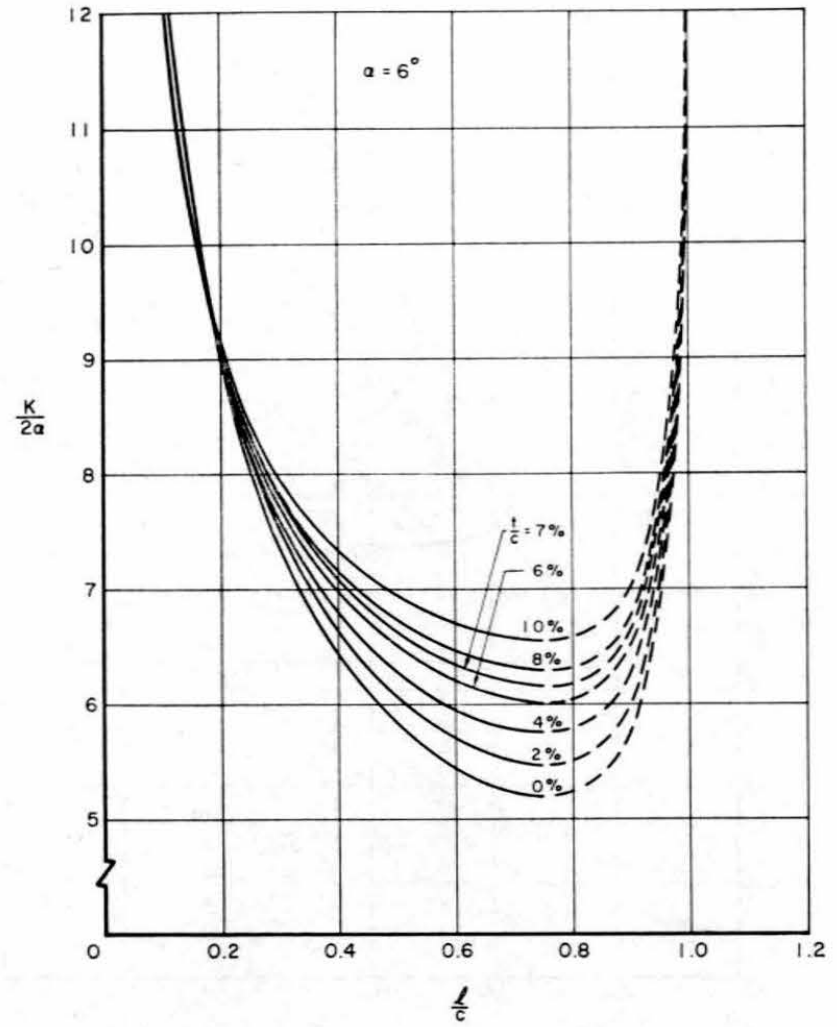

Fig. 5 Ratio of cavitation number to twice angle of attack as a function of cavity length-to-chord ratio for various thickness ratios at a fixed angle of attack of $6 \mathrm{deg}$

$$
\int_{\text {body }} d y=\int_{\text {body }} v d x=0
$$

or

$$
\operatorname{Im} \int_{\text {body }} W(\zeta) \frac{d z}{d \zeta} d \zeta=0
$$

where Im denotes the imaginary part of the integral. We are furthermore interested in the lift on the body. Within the limitations of the linearized theory this is given by

$$
C_{L}=-\frac{2}{V} \int_{\text {body }} u d x
$$

or

$$
C_{L}=-\frac{2}{V} \operatorname{Re} \int_{\text {body }} W(\zeta) \frac{d z}{d \zeta} d \zeta
$$

where Re denotes the real part of the integral. Since the body is now the entire real axis in the $\zeta$-plane, we have to evaluate the integral

$$
I=\int_{+\infty}^{-\infty} W(\zeta) \frac{d z}{d \zeta} d \zeta
$$

where

$$
\frac{d z}{d \zeta}=\frac{2 a^{2} \zeta}{(\zeta-i a)^{2}(\zeta+i a)^{2}}
$$

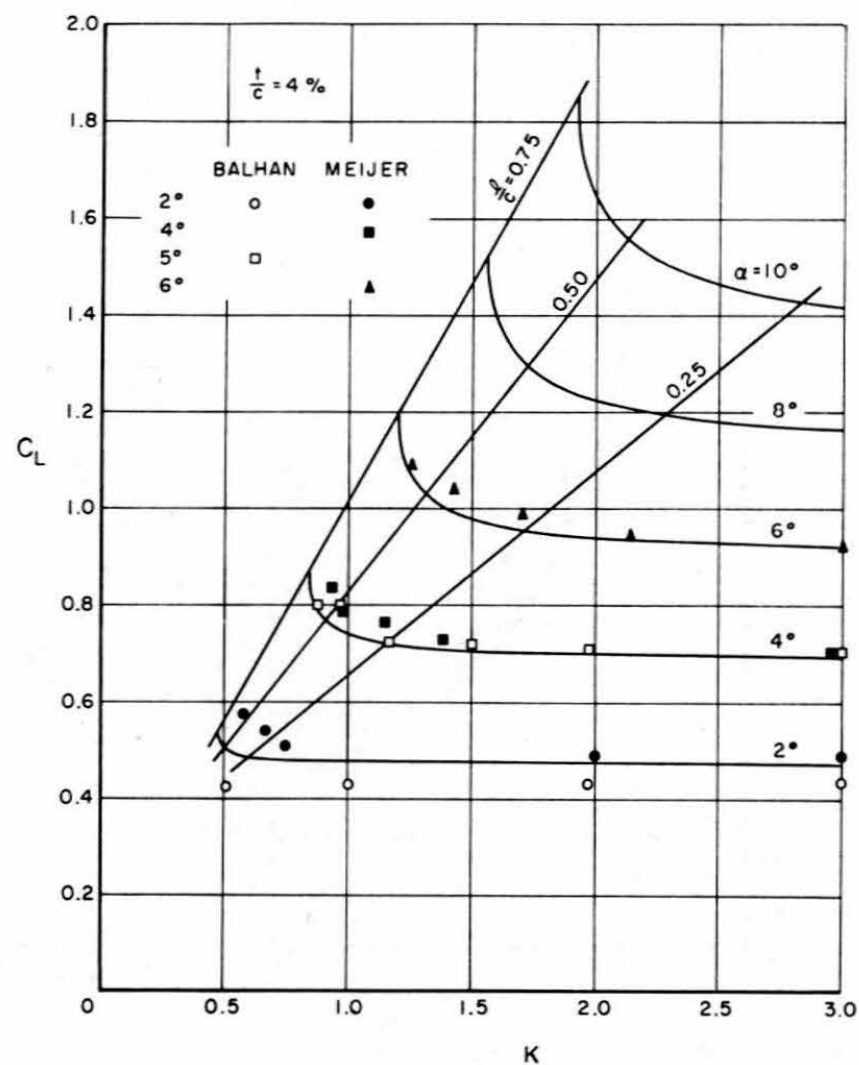

Fig. 6 Comparison of theoretical lift coefficient with experimental results for a 4-percent-thick plano-convex hydrofoil as a function of cavitation number for various angles of attack

Since

$$
\frac{d z}{d \zeta} \sim \frac{1}{\zeta^{3}} \text { as } \zeta \rightarrow \infty
$$

there is no contribution to the integral by completing the contour by a large circle $R$ and letting $R \rightarrow \infty$. The value of the integral is then given by the residue of the integrand at the double pole $\zeta=i a$. Since the integral traverses the body in a counter-clockwise fashion in the $z$-plane which corresponds to a clockwise sense in the $\zeta$-plane, we have

$$
I=-2 \pi i[\text { Residue at } \zeta=i a]
$$

which reduces to

$$
I=-\pi a\left[\frac{d W}{d \zeta}\right]_{\zeta=i a}
$$

Carrying out the indicated procedure and separating the result into real and imaginary parts we obtain, after considerable algebra, the following expressions for the closure condition and for the lift coefficient, $C_{L}$ :

$$
\begin{aligned}
& \frac{\pi A}{2 V a^{1 / 2}\left(1+a^{2}\right)^{3 / 4}} \cos \frac{3 \psi}{2} \\
& \quad+\frac{\pi B}{2 V a^{3 / 2}\left(1+a^{2}\right)^{3 / 4}}\left[\sin \frac{3 \psi}{2}+2 a \sin \frac{3 \psi}{2}\right]+
\end{aligned}
$$




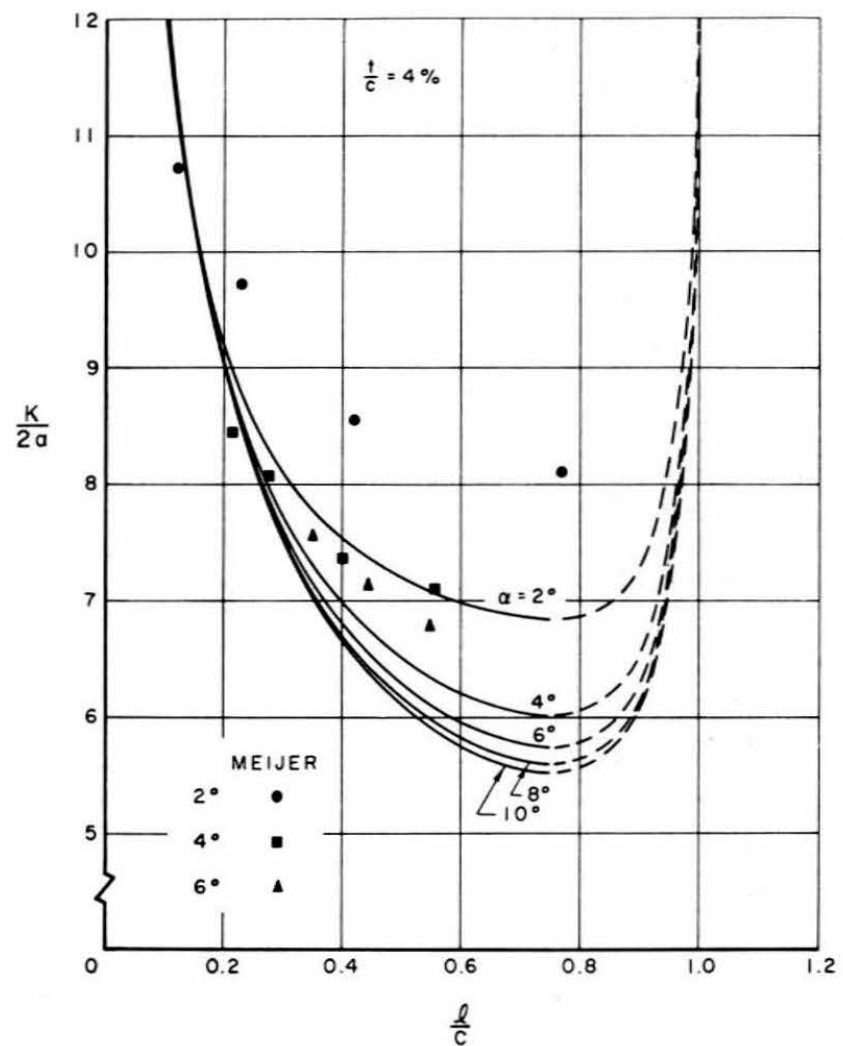

Fig. 7 Some cavity-length measurements compared with the theory for a 4-percent-thick plano-convex hydrofoil at various angles of attack

$$
\begin{aligned}
+\frac{\Omega}{2} a^{1 / 2}\left(1+a^{2}\right)^{1 / 4} & {\left[\left(\frac{1}{a} I_{2}-I_{4}\right) \sin \frac{\psi}{2}+I_{3} \cos \frac{\psi}{2}\right] } \\
+\frac{\Omega}{4} \frac{1}{a^{1 / 2}\left(1+a^{2}\right)^{1 / 4}} & {\left[I_{1}\left(\sin \frac{\psi}{2}+2 a \cos \frac{\psi}{2}\right)\right.} \\
& \left.-I_{2}\left(\cos \frac{\psi}{2}-2 a \sin \frac{\psi}{2}\right)\right]=0
\end{aligned}
$$

and

$$
\begin{aligned}
C_{L}=- & \frac{\pi A a^{1 / 2}}{V\left(1+a^{2}\right)^{3 / 4}} \sin \frac{3 \psi}{2} \\
+ & \frac{\pi B}{V a^{1 / 2}\left(1+a^{2}\right)^{3 / 4}}\left[\cos \frac{3 \psi}{2}-2 a \sin \frac{3 \psi}{2}\right] \\
-\Omega a^{3 / 2}\left(1+a^{2}\right)^{1 / 4}\left[\left(\frac{1}{a} I_{2}-I_{4}\right) \cos \frac{\psi}{2}-I_{3} \sin \frac{\psi}{2}\right] & -\frac{a^{1 / 2}}{2}\left[I_{1}\left(\cos \frac{\psi}{2}-2 a \sin \frac{\psi}{2}\right)\right. \\
+ & \left.\quad+I_{2}\left(\sin \frac{\psi}{2}+2 a \cos \frac{\psi}{2}\right)\right]
\end{aligned}
$$

where

$$
I_{3}=4 a \int_{0}^{1} \frac{t^{2}\left(a^{2} t^{2}-1\right)}{\left(1+a^{2} t^{2}\right)^{3}} d x
$$

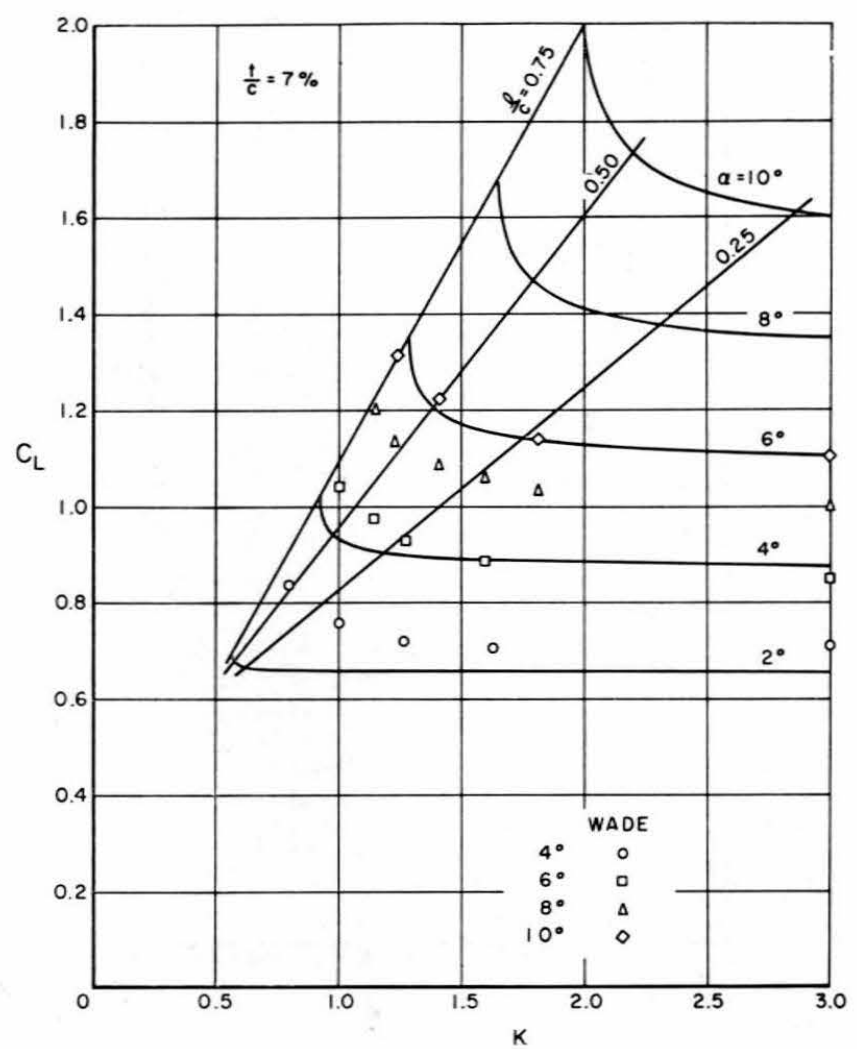

Fig. 8 Comparison of theoretical lift coefficient with experimental results for a 7-percent-thick plano-convex hydrofoil as a

function of cavitation number for various angles of attack

$$
I_{4}=4 a^{2} \int_{0}^{1} \frac{t^{3}\left(a^{2} t^{2}-1\right)}{\left(1+a^{2} t^{2}\right)^{3}} d x
$$

From these results the graphs shown in Figs. 4-10 were obtained. The computations were carried out on a IBM 7094 computer. The range of values investigated was for angles of attack from 2 to $10 \mathrm{deg}$ and values of $\Omega$ corresponding to thickness ratios of from 2 to 10 percent.

\section{Discussion}

Figs. 4 and 5 show the effect of profile contour on the performance of the hydrofoil under partially cavitating conditions. Only curves for one angle of attack, $\alpha=6$ deg, are shown. It is seen that the effects of camber and thickness are to increase lift at any given cavitation number with a corresponding increase in cavity length.

In the linearized theory the validity of the results is usually restricted to a certain range of cavitation numbers due to the type of cavity closure used. For the present case this range of validity holds for values of cavity length-to-chord ratio less than about 0.75 , the same value as for the flat-plate case $[3,4]$. This is apparent from Fig. 5 where it is seen that the cavitation number reaches a minimum value at this point. Values outside this range would give rise to the possibility of two cavity lengths for any given cavitation number.

It should be noted that the foregoing calculations are 


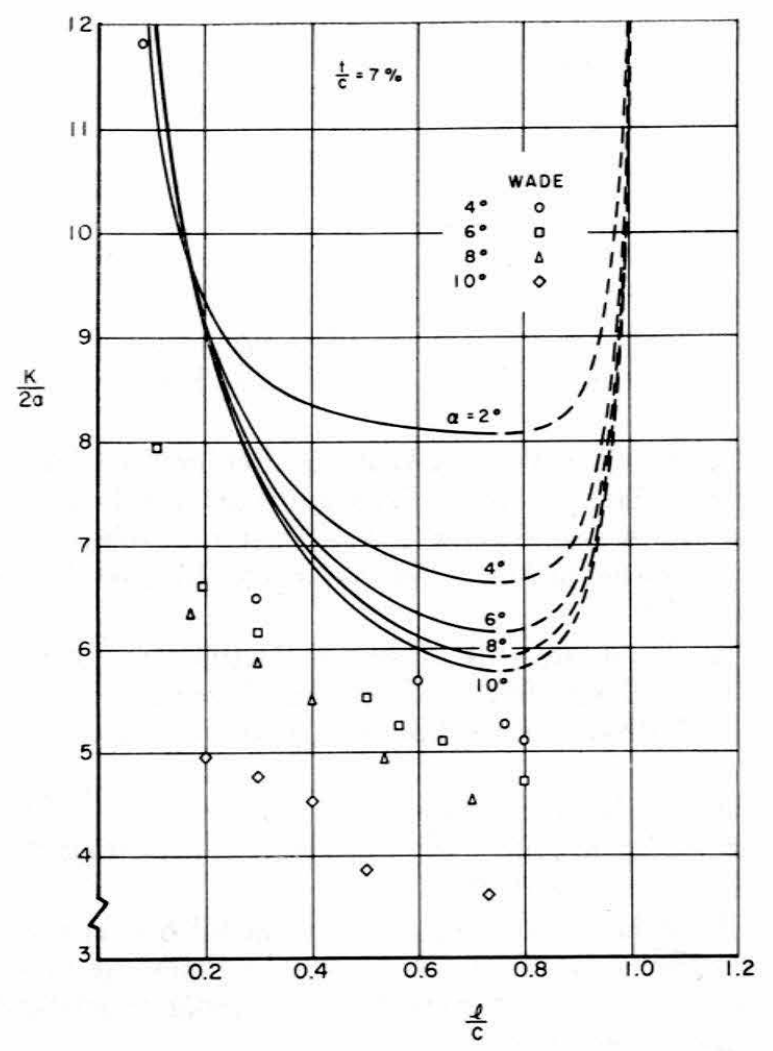

Fig. 9 Some cavity-length measurements compared with theory for a 7-percent-thick plano-convex hydrofoil at various angles of attack

based on the assumption that the cavity springs from the leading edge at all times. Under certain configurations of angle of attack and thickness ratio this assumption may not be physically possible, as brought out by the findings of experimental investigation [1], where at lower angles of attack a cavity starts downstream of the leading edge at approximately the point of maximum thickness. This should therefore be kept in mind when applying the aforementioned results.

It may be noted that the fully wetted results obtained from this linearized theory for a hydrofoil of 7 percent thickness ratio give a zero lift angle of attack of $-4 \mathrm{deg}$ with a corresponding lift coefficient at zero angle of attack of 0.438 . This compares with values of $-4 \mathrm{deg} 10$ min and 0.479 obtained by conformal mapping techniques, and with $-4 \mathrm{deg}$ and 0.38 obtained from experiment [1].

In Figs. 6 to 10 a comparison of the theoretical results is shown with points obtained from various experimental investigations. Fig. 6 illustrates the lift coefficients for a 4-percent-thick plano-convex hydrofoil as obtained by Balhan and Meijer. It will be seen that good agreement is found between experiment and theory. Fig. 7 compares some cavity-length measurements obtained by Meijer with those predicted by the present theory. Here the agreement is not as good.

The discrepancy in this case may be partly due to the

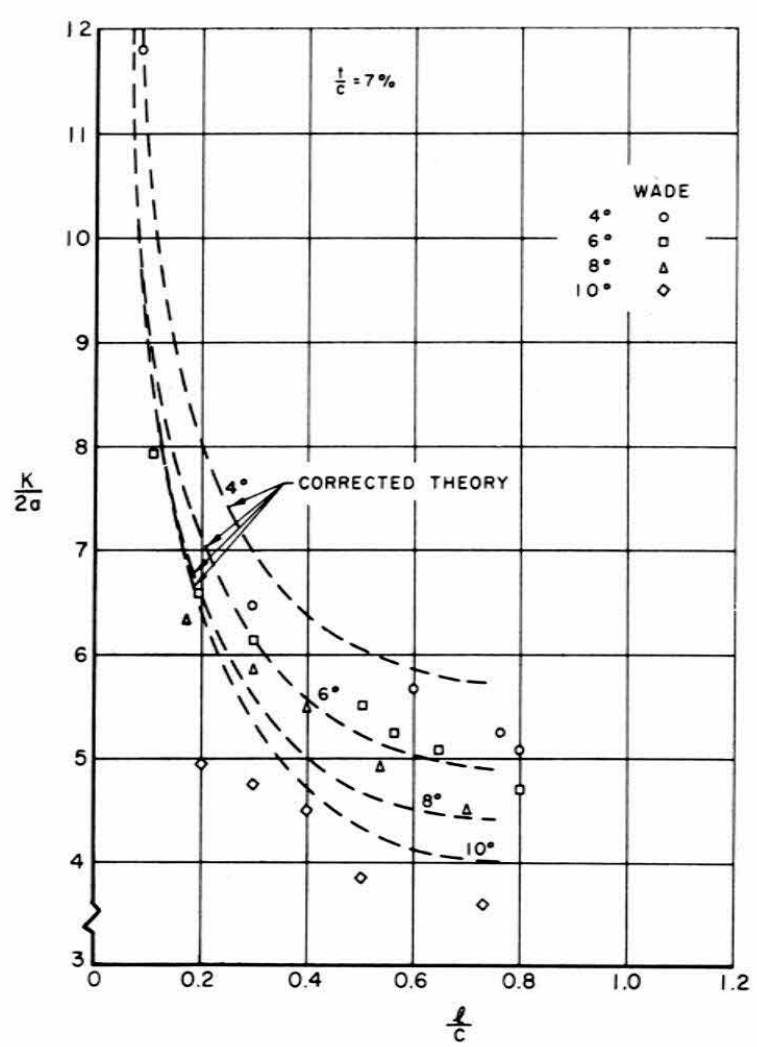

Fig. 10 Comparison of experimental cavity-length measurements with corrected theory for a 7 -percent-thick hydrofoil at various angles of attack

difficulty in measuring cavity lengths under these circumstances owing to a certain arbitrariness in interpreting where the cavity ends. However, a more likely explanation is the fact that in Meijer's experiments the cavitation numbers are based on vapor pressure and not on measured cavity pressure. It is well known that the cavitation number is decreased if measured cavity pressure is used in determining this parameter. This lowering would therefore tend to improve the correlation between experiment and theory.

In Fig. 8 comparison is made between experiment and theory for a 7-percent-thick hydrofoil. Here it will be seen that the experimental results are between 20 to 30 percent lower than the theoretical values - the larger value corresponding to the largest angle of attack. This discrepancy is probably due to the deterioration of the linearization at these higher thicknesses in conjunction with the increasing angle of attack. The corresponding cavity lengths are shown in Fig. 9 where again a similar discrepancy exists.

It is known from airfoil experiments that if the theoretical lift is arbitrarily adjusted to the experimental value, the theoretical pressure distribution on the foil agrees on the whole with the experimental one. This artifice achieves two purposes. First, it endeavors to some extent to account for real fluid effects and second, it affords a means of checking whether the experimental 
data are self-consistent. This approach was utilized here. The theoretical lift coefficient was adjusted to the experimental value for the same cavity lengths and the corresponding theoretical cavitation number was altered accordingly. These adjusted cavitation numbers are shown plotted in Fig. 10. It is seen that the corrected theoretical values are in good agreement with the experimental points.

The limiting case when the thickness of the hydrofoil is Zero reduees in the limit to that of the performance of a partially cavitating flat plate.

\section{Conclusion}

In conclusion it may be stated that the linearized theory presented predicts with sufficient accuracy the performance of a partially cavitating plano-convex hydrofoil having percentage thicknesses up to 5 percent. For larger thicknesses the linearization breaks down and for 7 percent thickness ratios overestimates the lift coefficients by 20 to 30 percent, for angles of attack up to $10 \mathrm{deg}$.

The limiting cases of a flat plate and a fully wetted plano-convex hydrofoil are retrieved by taking the appropriate limits.

\section{Acknowledgments}

The author wishes to thank Dr. A. J. Acosta for his constant interest and many helpful discussions. This work was supported by the Department of the Navy under Contract Nonr 220(24).

\section{References}

1 R. B. Wade and A. J. Acosta, "Experimental
Observations on the Flow Past a Plano-Convex Hydrofoil," Trans. ASME, Journal of Basic Engineering, Paper presented June 7, 1965, Applied Mechanics/Fluids Engineering Conference, Washington, D. C.

2 T. Yao-Tsu Wu and D. P. Wang, "A Wake Model for Free Streamline Flow Theory, Part 2, Cavity Flows Past Obstacles of Arbitrary Profile," Journal of Fluid Mechanics, vol. 18, part 1, 1964, pp. 65-93.

3 B. R. Parkin, "Linearized Theory of Cavity Flow in Two Dimensions," Rand Corporation Report P-1745, 1959.

4 T. Yao-Tsu Wu, "A Wake Model for Free-Streamline Flow Theory, Part 1, Fully and Partially Developed Wake Flows and Cavity Flows Past an Oblique Flat Plate," Journal of Fluid Mechanics, vol. 13, part 2, 1962, pp. 161-181.

5 A. J. Acosta, "A Note on Partial Cavitation of Flat Plate Hydrofoils," California Institute of Technology Hydrodynamics Laboratory Report No. E-19.9, 1955 .

6 J. A. Geurst, "Linearized Theory for Partially Cavitated Hydrofoils," International Shipbuilding Progress, vol. 6, no. 60, 1959 .

7 J. Balhan, "Metingen aan Enige bij Scheepschroenen Gebruikelijke Profielen in Vlokke Stroming met en Zonder Cavitie," Ned. Scheepsbouwkundig Proefstation te Wageningen, 1951.

8 M. C. Meijer, "Some Experiments on Partially Cavitating Hydrofoils," International Shipbuilding Progress, vol. 6 , no. 60,1959 .

9 N. I. Muskhelishvili, Singular Integral Equations, P. Noordhoff, Limited, Groningen, Holland, 1946. 